\title{
RECIST Progressive Disease
}

National Cancer Institute

\section{Source}

National Cancer Institute. RECIST Progressive Disease. NCI Thesaurus. Code C159716.

At lease a $20 \%$ increase in the sum of the longest diameter of target lesions, taking as reference the smallest sum longest diameter recorded since the treatment started or the appearance of one or more new lesions. 